\title{
UNIFORM INTEGRABILITY AND THE POINTWISE ERGODIC THEOREM
}

\author{
YUJI ITO
}

Let $(X, Q, m)$ be a finite measure space. We shall denote by $L^{p}(m)(1 \leqq p<\infty)$ the Banach space of all real-valued $B$-measurable functions $f$ defined on $X$ such that $|f|^{p}$ is $m$-integrable, and by $L^{\infty}(m)$ the Banach space of all real-valued, $B$-measurable, $m$-essentially bounded functions defined on $X$; as usual, the norm in $L^{p}(m)$ is given by $\|f\|_{p}=\left\{\int_{X}|f(x)|^{p} d m\right\}^{1 / p}$, and the norm in $L^{\infty}(m)$ by $\|g\|_{\infty}$ $=m$-ess. $\sup _{x \in X}|g(x)|$. Two functions in $L^{p}(m)$ or $L^{\infty}(m)$ will be identified if they differ only on a set of $m$-measure zero. In this note, we shall be concerned with a positive linear operator $T$ of $L^{1}(m)$ into $L^{1}(m)$ with $\|T\|_{1} \leqq 1$.

We say that the pointwise ergodic theorem (the $L^{1}(m)$-mean ergodic theorem, respectively) holds for such an operator $T$ if for every $f$ in $L^{1}(m)$, the sequence of averages $\left\{1(/ n) \sum_{k=0}^{n-1} T^{k} f\right\}$ converges $m$-almost everywhere (in the norm of $L^{1}(m)$, respectively) to a function in $L^{1}(m)$. Recently, R. V. Chacon [1] constructed a class of positive linear operators in $L^{1}(m)$ with the norm equal to 1 for which the pointwise ergodic theorem fails to hold. Also, A. Ionescu Tulcea [5], [6] showed that in the group of all positive invertible linear isometries of $L^{1}(m)$ the set of all $T$ for which the pointwise ergodic theorem fails to hold forms a set of second category with respect to the strong operator topology. On the other hand, the ergodic theorem of HopfDunford-Schwartz [4] tells us that if, in addition, $T$ maps $L^{\infty}(m)$ into $L^{\infty}(m)$ and $\|T\|_{\infty} \leqq 1$, then the pointwise ergodic theorem is valid for such $T$. In view of these facts, it is interesting to find out what other additional conditions on $T$ would guarantee the validity of the pointwise ergodic theorem. In this note, we shall find a few such conditions which are weaker than the condition of the Hopf-Dunford-Schwartz theorem (though our conditions seem to work for a finite measure space only). We also obtain a result (corollary to Theorem 1, below) which generalizes a result obtained by N. Dunford and D. S. Miller in $[3]$.

First of all, let us observe that if our operator $T$ satisfies $\|T\|_{1}<1$, then the pointwise ergodic theorem is always valid. This is because, for such an operator $T, \sum_{n=0}^{\infty}\left|T^{n} f(x)\right|<\infty m$-almost everywhere for every $f$ in $L^{1}(m)$, since

Received by the editors September 26, 1963. 


$$
\int_{X} \sum_{n=0}^{\infty}\left|T^{n} f(x)\right| d m=\sum_{n=0}^{\infty}\left\|T^{n}\right\|_{1} \leqq\|f\|_{1} \cdot \sum_{n=0}^{\infty}\|T\|_{1}^{n}=\frac{\|f\|_{1}}{1-\|T\|_{1}}<\infty .
$$

Therefore, we shall assume from now on that $T$ satisfies $\|T\|_{1}=1$.

Let us denote by $U$ the adjoint operator of $T$. Then, $U$ is a linear operator mapping $L^{\infty}(m)$ into $L^{\infty}(m)$, and for every pair of functions $f, g$ with $f$ in $L^{1}(m), g$ in $L^{\infty}(m)$,

$$
\int_{\mathbf{X}} g(x) T f(x) d m=\int_{\mathbf{X}} f(x) U g(x) d m
$$

holds. It is clear that $U$ is also a positive operator and that $\|U\|_{\infty}=\|T\|_{1}=1$.

We say that a sequence of functions $\left\{f_{n}\right\}$ in $L^{1}(m)$ is uniformly integrable if

$$
\lim _{N \rightarrow \infty} \int_{\left\{\left|f_{n}(x)\right|>N\right\}}\left|f_{n}(x)\right| d m=0 \text { uniformly in } n .
$$

A subset $K$ of a Banach space $E$ is called weakly sequentially compact if every sequence $\left\{\phi_{n}\right\}$ of elements in $K$ contains a subsequence which converges weakly to an element in $E$. It is well known that a sequence $\left\{f_{n}\right\}$ in $L^{1}(m)$ is uniformly integrable if and only if the set $\left\{f_{n}\right\}$ is weakly sequentially compact in $L^{1}(m)$.

We shall need the following

LEMMA 1. Let $\left\{f_{n}\right\}$ and $\left\{g_{n}\right\}$ be two sequences of functions in $L^{1}(m)$ such that, for each $n,\left|g_{n}(x)\right| \leqq\left|f_{n}(x)\right|$ holds m-almost everywhere. Suppose $\left\{f_{n}\right\}$ is uniformly integrable, then so is $\left\{g_{n}\right\}$.

Proof. Obvious from the following inequalities

$$
\int_{\left\{\left|O_{n}(x)\right|>N\right\}}\left|g_{n}(x)\right| d m \leqq \int_{\left\{\left|O_{n}(x)\right|>N\right\}}\left|f_{n}(x)\right| d m \leqq \int_{\left\{\left|f_{n}(x)\right|>N\right\}}\left|f_{n}(x)\right| d m .
$$

The main result of this note is the following

THEOREM 1. Suppose for our operator $T$, the sequence of functions $\left\{(1 / n) \sum_{k=0}^{n-1} T^{k} 1\right\}$, where $1(x)$ denotes the function taking constant value one m-almost everywhere, is uniformly integrable; then, the pointwise ergodic theorem holds for $T$.

For the proof of this theorem, we shall need a few more lemmas.

LEMMA 2. Suppose the hypothesis of Theorem 1 is satisfied for $T$, then the $L^{1}(m)$-mean ergodic theorem holds for $T$. 
Proof. Let, for any set $B$ in $B, \chi_{B}(x)$ denote its characteristic function. By the positivity of $T$, we have $0 \leqq T^{k} \chi_{B}(x) \leqq T^{k} 1(x) m$-almost everywhere for each $k$. Therefore, for each $n$,

$$
0 \leqq \frac{1}{n} \sum_{k=0}^{n-1} T^{k} \chi_{B}(x) \leqq \frac{1}{n} \sum_{k=0}^{n-1} T^{k} 1(x)
$$

holds $m$-almost everywhere. Since the set of all characteristic functions of sets in $B$ forms a fundamental subset of $L^{1}(m)$, Lemma 1 and our hypothesis imply that the set $\left\{(1 / n) \sum_{k=0}^{n-1} T^{k} f\right\}$ is weakly sequentially compact in $L^{1}(m)$ for every $f$ belonging to a fundamental subset of $L^{1}(m)$. Since it is clear that $\left\|(1 / n) \sum_{k=0}^{n-1} T^{k}\right\|_{1} \leqq 1$ for all $n$, the mean ergodic theorem of Yosida and Kakutani [7] now implies that the $L^{1}(m)$-mean ergodic theorem must hold for our T. Q.E.D.

Suppose now the $L^{1}(m)$-mean ergodic theorem is valid for $T$. Let us write

$$
h=s-\lim \frac{1}{n \rightarrow \infty} \sum_{k=0}^{n-1} T^{k} 1
$$

where $s$-lim means the limit taken in the sense of the norm in $L^{1}(m)$. Then, it is clear that

(i) $h \in L^{1}(m)$,

(ii) $h(x) \geqq 0 m$-almost everywhere,

(iii) $T h(x)=h(x)$ holds $m$-almost everywhere (i.e., $h$ is invariant under $T)$.

Furthermore, since the norm-convergence implies the weak convergence, we have

(iv) for every set $B$ in $B$,

$$
\int_{B} h(x) d m=\lim _{n \rightarrow \infty} \frac{1}{n} \sum_{k=0}^{n-1} \int_{B} T^{k} 1(x) d m=\lim _{n \rightarrow \infty} \frac{1}{n} \sum_{k=0}^{n-1} \int_{X} U^{k} \chi_{B}(x) d m .
$$

Lemma 3. Let us denote $A=\{x \mid h(x)=0\}$, where $h(x)$ is the function defined above. Then, $U \chi_{A}(x) \leqq \chi_{A}(x)$ holds $m$-almost everywhere.

Proof. Since $U$ satisfies $\|U\|_{\infty}=1$, it follows that $U 1(x) \leqq 1$ $m$-almost everywhere. Now, the positivity of $U$ implies $U \chi_{A}(x)$ $\leqq U 1(x) \leqq 1$ holds $m$-almost all $x$. Therefore, for $m$-almost all $x$ in $A$, $U \chi_{A}(x) \leqq \chi_{A}(x)$ is satisfied. Now, suppose $x \in X-A$. We must prove that $U \chi_{A}(x)=0$ holds for $m$-almost all such $x$. Suppose this were not the case. Then, there would exist a set $B \subset X-A$ such that $m(B)>0$ and such that $U \chi_{A}(x)>0$ holds for all $x$ in $B$. But then, since $h(x)>0$ for all $x \in B$ and $h(x) U \chi_{A}(x) \geqq 0$ for $m$-almost all $x \in X$, we get 


$$
\begin{aligned}
0 & <\int_{B} h(x) U \chi_{A}(x) d m \leqq \int_{X} h(x) U \chi_{A}(x) d m=\int_{A} T h(x) d m \\
& =\int_{A} h(x) d m=0
\end{aligned}
$$

a contradiction. Q.E.D.

Since $U$ is order preserving, Lemma 3 implies that

$$
\chi_{A}(x) \geqq U \chi_{A}(x) \geqq \cdots \geqq U^{k} \chi_{A}(x) \geqq \cdots
$$

holds $m$-almost everywhere for the set $A$.

Lemma 4. Let $A$ be the same set as in Lemma 3. Then,

$$
A \subset \bigcup_{k=1}^{\infty}\left\{x \mid U^{k} \chi_{A}(x)<\chi_{A}(x)\right\}
$$

with the possible exception of a set of m-measure zero.

Proof. Suppose false. Then, there would exist a set $C \subset A$ such that $m(C)>0$ and such that $U^{k} \chi_{A}(x) \geqq \chi_{A}(x)$ holds for all $x$ in $C$ and for all $k$. Since $U^{k} \chi_{A} \leqq \chi_{A}(x)$ must hold $m$-almost everywhere for each $k$, this means we have $U^{k} \chi_{A}(x)=\chi_{A}(x)=1$ for all $k$ and for $m$-almost all $x$ in $C$. But then, for each $n$,

$$
\begin{aligned}
0 & <m(C)=\frac{1}{n} \sum_{k=0}^{n-1} \int_{C} d m=\frac{1}{n} \sum_{k=0}^{n-1} \int_{C} U^{k} \chi_{A}(x) d m \\
\leqq & \frac{1}{n} \sum_{k=0}^{n-1} \int_{X} U^{k} \chi_{A}(x) d m=\frac{1}{n} \sum_{k=0}^{n-1} \int_{A} T^{k} 1(x) d m,
\end{aligned}
$$

and therefore,

$$
\lim _{n \rightarrow \infty} \frac{1}{n} \sum_{k=0}^{n-1} \int_{A} T^{k} 1(x) d m \geqq m(C)>0 .
$$

But, since the left side of the last inequality equals $\int_{A} h(x) d m=0$, we have a contradiction.

Lemma 5. For every $f$ in $L^{1}(m), \lim _{n \rightarrow \infty}(1 / n) \sum_{k=0}^{n-1} T^{k} f(x)=0$ $m$-almost everywhere on the set $A$.

Proof. Actually, we can prove even more: on the set $A$, $\sum_{n=0}^{\infty} T^{n}|f|(x)<\infty$ holds $m$-almost everywhere for every $f$ in $L^{1}(m)$. In view of Lemma 4 , it suffices to prove this fact on the set $\left\{x \mid U^{k} \chi_{A}(x)<\chi_{A}(x)\right\}$ for each fixed integer $k$. So, let us fix $k$. Then, for any $n>k$, we have 


$$
\begin{aligned}
0 & \leqq \int_{X}\left(\sum_{j=0}^{n-1} T^{j}|f|(x)\right)\left(\chi_{A}(x)-U^{k} \chi_{A}(x)\right) d m \\
& =\int_{X}|f|(x)\left\{\sum_{j=0}^{n-1} U^{j}\left(\chi_{A}-U^{k} \chi_{A}\right)(x)\right\} d m \\
& =\int_{X}|f|(x)\left\{\chi_{A}(x)+U \chi_{A}(x)+\cdots+U^{k-1} \chi_{A}(x)-U^{n} \chi_{A}(x)-\cdots\right. \\
& \leqq 2 k \int_{X}|f|(x) d m<\infty
\end{aligned}
$$

Since this is true for any $n>k$, we must have $\sum_{\substack{\infty=0 \\ \infty}}^{\infty} T^{j}|f|(x)<\infty$ $m$-almost everywhere on the set

$$
\left\{x \mid U^{k} \chi_{A}(x)<\chi_{A}(x)\right\} . \quad \text { Q.E.D. }
$$

Proof of Theorem 1. By Lemma 2, we know that there exists a non-negative function $h(x)$ which is invariant under $T$. For any $f$ in $L^{1}(m)$, we now apply the general ergodic theorem of Chacon and Ornstein [2] to $f$ and $h$. Then,

$$
\lim _{n \rightarrow \infty} \frac{\sum_{k=0}^{n-1} T^{k} f}{\sum_{k=0}^{n-1} T^{k} h}=\frac{1}{h} \lim _{n \rightarrow \infty} \frac{1}{h} \sum_{k=0}^{n-1} T^{k} f
$$

exists $m$-almost everywhere on the set $\{x \mid h(x)>0\}$. Therefore, $\lim _{n \rightarrow \infty}(1 / n) \sum_{k=0}^{n-1} T^{k} f(x)$ exists $m$-almost everywhere on the set $\{x \mid h(x)>0\}$ for every $f$ in $L^{1}(m)$. On the other hand, by Lemma 5 , $\lim _{n \rightarrow \infty}(1 / n) \sum_{k=0}^{n-1} T^{k} f(x)=0 m$-almost everywhere on $\{x \mid h(x)=0\}$ for every $f$ in $L^{1}(m)$. Thus, the pointwise ergodic theorem is valid for T. Q.E.D.

REMARK. If in Theorem $1, f$ in $L^{1}(m)$ is strictly positive $m$-almost everywhere, then $\lim _{n \rightarrow \infty}(1 / n) \sum_{k=0}^{n-1} T^{k} f(x)$ is strictly positive $m$ almost everywhere on the set $\{x \mid h(x)>0\}$, because if not, the limit of the ratio

$$
\frac{\sum_{k=0}^{n-1} T^{k} h(x)}{\sum_{k=0}^{n-1} T^{k} f(x)}
$$


would become unbounded on some part of the set $\{x \mid h(x)>0\}$, contradicting the general ergodic theorem of Chacon-Ornstein.

The argument used in the proof above includes the following

CoROLlaRY. If the $L^{1}(m)$-mean ergodic theorem holds for $T$, then the pointwise ergodic theorem is also true for $T$.

We can also prove

THEOREM 2. Suppose $T$ satisfies the following additional condition: for some $p, 1<p<\infty, T$ maps $L^{p}(m)$ into $L^{p}(m)$ and $\|T\|_{p} \leqq 1$. Then, the pointwise ergodic theorem is valid for $T$ in $L^{1}(m)$.

Proof. Since $L^{p}(m), 1<p<\infty$, is reflexive, any bounded subset is weakly sequentially compact. Therefore, the set $\left\{(1 / n) \sum_{k=0}^{n-1} T^{k} 1\right\}$ is weakly sequentially compact in $L^{p}(m)$. Since $(X, B, m)$ is a finite measure space, this implies that $\left\{(1 / n) \sum_{k=0}^{n-1} T^{k} 1\right\}$ is weakly sequentially compact in $L^{1}(m)$ as well. Therefore, the proof of Theorem 2 is reduced to that of Theorem 1 .

REMARK. Suppose as in the Hopf-Dunford-Schwartz theorem, $T$ maps $L^{\infty}(m)$ into $L^{\infty}(m)$ and $\|T\|_{\infty} \leqq 1$. Then, for each $n$,

$$
0 \leqq \frac{1}{n} \sum_{k=0}^{n-1} T^{k} 1(x) \leqq 1 \quad m \text {-almost everywhere }
$$

so that it is clear $\left\{(1 / n) \sum_{k=0}^{n-1} T^{k} 1\right\}$ is uniformly integrable in this case since $m(X)<\infty$. Thus, the hypothesis of our Theorem 1 is weaker than that of Hopf-Dunford-Schwartz. Also, by the Riesz convexity theorem, $\|T\|_{1} \leqq 1,\|T\|_{\infty} \leqq 1$ would imply that $T$ maps $L^{p}(m)$ into $L^{p}(m)$ for each $p, 1<p<\infty$, and $\|T\|_{p} \leqq 1$. Therefore, the hypothesis of our Theorem 2 is also weaker.

\section{BIBLIOGRAPHY}

1. R. V. Chacon, $A$ class of linear transformations, Proc. Amer. Math. Soc. 15 (1964), 560-564.

2. R. V. Chacon and D. S. Ornstein, A general ergodic theorem, Illinois J. Math. 4 (1960), 153-160.

3. N. Dunford and D. S. Miller, On the ergodic theorem, Trans. Amer. Math. Soc. 60 (1946), 538-549.

4. N. Dunford and J. T. Schwartz, Linear operators, Vol. I, Interscience, New York, 1957.

5. A. Ionescu Tulcea, Un theorème de categorie dans la theorie ergodique, C. R. Acad. Sci. Paris 257 (1963), 18-20.

6. - On the category of certain classes of transformations in ergodic theory, Trans. Amer. Math. Soc. 114 (1965), 261-279.

7. K. Yosida and S. Kakutani, Operator-theoretical treatment of Markoff's process and mean ergodic theorem, Ann. of Math. (2) 42 (1941), 188-228.

Brown UNIVERSITY 